\title{
Electromechanical delay detected by tissue Doppler echocardiography is associated with the frequency of attacks in patients with lone atrial fibrillation
}

\author{
Ali Nazmi Çalık, Kazım Serhan Özcan, Metin Çağdaş, Barış Güngör, \\ Gürkan Karaca, Ufuk Gürkan, Hale Y1lmaz, Osman Bolca \\ Department of Cardiology, Siyami Ersek Cardiovascular and Thoracic Surgery Center, Istanbul, Turkey
}

\begin{abstract}
Background: Our main purpose in this study is to compare atrial (inter-atrial, intra-left atrial, intra-right atrial) electromechanical delays of patients with lone atrial fibrillation (LAF) with healthy individuals and examine the relationship of annual LAF attack frequency.

Methods: 32 entirely healthy individuals and 32 patients who have presented with tachycardia and complying with LAF criteria have been included in the study. The time passing from the beginning of the P wave on electrocardiography to the A' wave on tissue Doppler trace was accepted as the atrial conduction time (PA'). The PA' time difference between the mitral annulus of left ventricle (ML) and the tricuspid annulus of right ventricle (TL) was defined as inter-atrial electromechanical delay (IA-EMD), the PA' time difference between the ML and septal mitral annulus (MS) as intra-left electromechanical delay (ILeft-EMD), the PA' time difference between MS and the TL as intra-right electromechanical delay (IRight-EMD).

Results: ILeft-EMD $(21.8 \pm 9.1$ vs. $14.1 \pm 4.9, p<0.001)$, IRight-EMD $(9.3 \pm 6.8$ vs. $5.9 \pm$ $\pm 4.9, p=0.03)$ and IA-EMD times $(24.7 \pm 11.2$ vs. $11.9 \pm 7.1, p<0.001)$ were significantly longer in LAF patients. In multivariate regression analysis, using a model including age, gender and left atrium (LA) volumes, ILeft-EMD times (OR 1.14, 95\% CI 1.03-1.27, $p=0.012)$, IA-EMD times (OR 1.12, 95\% CI 1.03-1.23, $p=0.007)$ and LA volumes (OR 1.18, 95\% CI 1.05-1.32, $p=0.005$ ) were independent predictors of LAF. In LAF group, the frequency of $A F$ episodes was significantly correlated with ILeft-EMD $(r=0.90, p<0.001)$ and IA-EMD times ( $r=0.36, p<0.004)$, whereas, IRight-EMD times and LA volumes were not correlated with recurrence rates.

Conclusions: ILeft-EMD and IA-EMD may increase in the early stages of atrial fibrillation even without the left atrial dilation and may be more valuable than left atrial area and volume in predicting atrial fibrillation. (Cardiol J 2014; 21, 2: 138-143)
\end{abstract}

Key words: atrial fibrillation, electromechanical delay, inter-atrial conduction time

Address for correspondence: Dr Ali Nazmi Çalık, Acıbadem Mah, Tekin Sokak, Erdem Sitesi, E Blok, Daire: 16, 34817, Kadikoy, Istanbul, Turkey, tel: +90 532 6742429, +90 21654547 36, fax: +90 216561 38 37, e-mail: calik_nazmi@hotmail.com Received: 17.04.2013 Accepted: 02.07.2013

It has been presented as a poster presentation in the ACC Congress 2013, in San Francisco, USA as "Electromechanical Delay Detected by Tissue Doppler Echocardiography Is Associated with the Frequency of Attacks in Patients with Lone Atrial Fibrillation" and the abstract was published in JACC, volume 61, issue 10. 


\section{Introduction}

Atrial fibrillation (AF) is the most commonly seen arrhythmia in clinical practice [1]. Lone AF (LAF) is a subtype of AF which is seen in patients aged under 60 years and with no clinical history or evidence of cardiovascular disease [2]. In clinical practice, the ratio of LAF cases presenting with paroxysmal $\mathrm{AF}$ and progression to permanent $\mathrm{AF}$ in long term follow-up is relatively low (30\% in 30 years), although it increases with age and comorbid diseases [3].

The mechanisms that trigger and make $\mathrm{AF}$ persistent are not fully understood today. It has been determined that ectopic activities arising from pulmonary veins play an important role in triggering $\mathrm{AF}$ [4]. It has also been proven that inter-atrial conduction abnormalities are important in the initiation and persistence of $\mathrm{AF}$ and that the frequency of $\mathrm{AF}$ attacks is associated with longer inter-atrial conduction delay times [5-7].

Determining electromechanical events including the inter-atrial conduction time with transthoracic echocardiography is a noninvasive and simple method [8]. However, there are no prospective studies proving that this method can be used to predict AF.

Our main purpose in this study is to compare inter-atrial delay times of the patients with LAF to the control group and examine the relationship of annual $\mathrm{AF}$ attack frequency with the determined atrial conduction and electromechanical delays times.

\section{Methods}

Thirty-two patients who have presented with tachycardia and complying with LAF criteria in Dr Siyami Ersek Cardiovascular and Thoracic Surgery and Research Hospital between January and August 2011 were included in the study. Patients with structural heart diseases, rheumatic heart diseases, cardiomyopathy, heart failure, hypertension, diabetes, thyroid dysfunction and those with persistent or postoperative AF were excluded. A detailed physical examination was performed to all participants. Basal electrocardiography (ECG) recordings and blood samples for complete blood count analysis and biochemical parameters were collected from all participants. The patient group was followed prospectively for 1 year and their paroxysmal AF attacks, documented with ECG and Holter ECG recordings, were collected. Thirty-two healthy individuals with the same demographic characteristics were included in the study as the control group. Written consent was obtained from all the patients and the study protocol was approved by the local ethics commitee of hospital.

All echocardiographic evaluations were performed using a transducer of 2.5-3.5 MHz and GE Vivid 3 echocardiography device. Each patient was evaluated with an M-mode, 2-dimensional Doppler and tissue Doppler echocardiography. Single lead ECG was recorded constantly during the workup. The end-systolic and diastolic diameters of the left ventricle (LV), as well as the end-systolic diameter of the left atrium (LA) were measured with M-mode echocardiography on the parasternal long axis display, in accordance with the American Society of Echocardiography guidelines [9]. The end-systolic LA area (largest area or the area calculated at the end of the T-wave) was measured on the apical 4 chamber view using the area length method. The average of the areas during last 3 consecutive beats was measured as the LA area. LA volume was calculated with the $(\mathrm{A} 1 \times \mathrm{A} 2 \times \mathrm{A} 3 \times 0.524)$ formula using the LA dimensions measured from the parasternal long axis (anteroposterior - A1) and apical 4 chamber (mediolateral - A2, apicobasal - A3) views. Mitral inflow velocities were evaluated by pulse-wave Doppler echocardiography with the sample volume placed at the tip of the mitral leaflets from the apical 4 chamber view. Diastolic peak early (E) and peak late (A) transmitral flow velocity, peak $\mathrm{E}$ to peak $\mathrm{A}$ velocities (E/A), and deceleration time of peak E velocity (DT) and isovolumic relaxation time (IVRT) were measured. The LV-pulsed tissue Doppler imaging was performed in the apical 4 chamber view using a 5-mm pulsed Doppler sample volume as minimum optimal gain to obtain the best signal to noise ratio. We adjusted the signal filter of the spectral pulsed Doppler until the Nyquist limit was $15-20 \mathrm{~cm} / \mathrm{s}$ using a transducer of 3.5-4.0 MHz in frequency and used minimal optimal gain to perform the tissue Doppler echocardiography. The monitor sweep speed was set at $50-100 \mathrm{~mm} / \mathrm{s}$ to optimize the spectral display of myocardial velocities. On the apical 4 chamber view, the pulsed wave Doppler cursor was placed on the lateral mitral annulus of the LV, septal mitral annulus and right ventricle's tricuspid annulus respectively, to obtain tissue Doppler spectral images. The myocardial peak systolic (S'), and peak early (E') and late diastolic (A') velocities were obtained from these areas. All echocardiographic measurements were performed by the same observer. The time passing from the beginning of the $\mathrm{P}$ wave on ECG to the A' wave 
Table 1. Comparison of the atrial fibrillation and control groups' demographical and clinical features.

\begin{tabular}{lccc}
\hline & Patient group $(\mathbf{n}=\mathbf{3 2})$ & Control group $(\mathbf{n}=32)$ & $\mathbf{P}$ \\
\hline Age [years] & $48.3 \pm 11.1$ & $45.2 \pm 4.8$ & 0.16 \\
Male gender & $23 \%$ & $20 \%$ & 0.42 \\
Atrial fibrillation type and frequency & \multicolumn{3}{l}{} \\
Paroxysmal atrial fibrillation & 32 & 0 \\
Attack number/year & $2.8 \pm 1.7$ & 0 & \\
Laboratory findings & & & 0.62 \\
Hemoglobin $[\mathrm{g} / \mathrm{dL}]$ & $14.8 \pm 1.7$ & $14.6 \pm 1.15$ & 0.92 \\
White blood cells $\left[10^{3} / \mu \mathrm{L}\right]$ & $6.62 \pm 1.88$ & $6.65 \pm 1.11$ & 0.12 \\
Thrombocytes $\left[10^{3} / \mu \mathrm{L}\right]$ & $267.4 \pm 48.2$ & $148.4 \pm 48.9$ & 0.10 \\
Mean platelet volume $[\mathrm{fL}]$ & $8.81 \pm 0.70$ & $9.16 \pm 0.93$ & 0.32 \\
Fasting glucose $[\mathrm{mg} / \mathrm{dL}]$ & $89.7 \pm 8.65$ & $91.7 \pm 7.23$ & 0.16 \\
Aspartate transaminase $[\mathrm{IU} / \mathrm{L}]$ & $25.7 \pm 7.6$ & $23.2 \pm 6.7$ & \\
\hline
\end{tabular}

on tissue Doppler trace was accepted as the atrial conduction time $\left(\mathrm{PA}^{\prime}\right)$. The $\mathrm{PA}^{\prime}$ time difference between the mitral annulus of left ventricle (ML) and the tricuspid annulus of right ventricle (TL) was defined as inter-atrial electromechanical delay (IA-EMD), the PA' time difference between the mitral annulus of left ventricle (ML) and septal mitral annulus (MS) as intra-left electromechanical delay (ILeft-EMD), the PA' time difference between septal mitral annulus (MS) and the tricuspid annulus of right ventricle (TL) as intra-right electromechanical delay (IRight-EMD).

\section{Statistical analyses}

All analyses were performed using the SPSS 16.0 software pack. The continuous variables within group data were calculated as average \pm \pm standard deviation and categorical variables as numbers and percentages. The t-test was used in groups that are independent of parametric tests and Mann-Whitney U-test as a non-parametric test for single-variable comparisons between the groups. $\mathrm{A} \chi^{2}$ test was used for comparing categorical variables. The correlations between single variables were evaluated with Pearson or Spearman correlation tests. The statistical significance reference was $\mathrm{p}<0.05$.

\section{Results}

Thirty-two patients with LAF and 32 healthy controls matching these patients' ages and genders were included in our study. The demographic and clinical features of patients are presented in Table 1. There were no significant differences regarding age, gender or risk factors such as hyperlipidemia, hypertension, thyroid dysfunction, diabetes or smoking between the two groups. The average attack frequency among the patient group was $2.8 \pm 1.7$ years. In the comparison of laboratory parameters of the two groups, no significant difference was found in white blood cells, hemoglobin, mean platelet volume, thrombocytes, glucose or aspartate transaminase levels (Table 1).

The comparative echocardiographic findings of the patients are presented in Table 2 . When the echocardiographic parameters were compared between the two groups, no significant difference was found regarding ventricular wall thicknesses, sizes and LV ejection fractions. However, the LA volume and LA area were found to be significantly higher in patients with LAF ( $<<0.001)$. Diastolic E and A velocities, DT and IVRT were not significantly different (Table 2).

In the evaluation of atrial conduction times, the atrial conduction times measured from the mitral valve's lateral (ML) and septal annulus (MS) were found to be higher in the patient group $(\mathrm{p}<0.001)$, whereas the conduction time measured from the tricuspid lateral annulus (TL) was not significantly different $(\mathrm{p}<0.28)$. Complying with these data, ILeft-EMD time and IA-EMD time were observed to be significantly higher in the patient group $(\mathrm{p}<0.001)$. IRight-EMD was also higher in the LAF group $(p=0.03)$ (Table 2, Fig. 1).

In multivariate regression analysis, using model including age, gender and LA volumes, ILeft-EMD times (OR 1.14, 95\% CI 1.03-1.27, p = $=0.012)$, IA-EMD times (OR 1.12,95\% CI1.03-1.23, $\mathrm{p}=0.007)$ and LA volumes (OR 1.18, 95\% CI $1.05-1.32, \mathrm{p}=0.005)$ were independent predictors of LAF. In the subgroup correlation analysis within LAF patients, the annual frequency of AF attacks 
Table 2. Comparison of conventional echocardiographic parameters and left atrial conduction times calculated with echocardiography.

\begin{tabular}{|c|c|c|c|}
\hline & Patient group ( $n=32$ ) & Control group $(n=32)$ & $\mathbf{P}$ \\
\hline LVEDd [mm] & $47.9 \pm 3.2$ & $46.9 \pm 2.9$ & 0.21 \\
\hline LVESd [mm] & $28.8 \pm 3.4$ & $28.4 \pm 3.2$ & 0.64 \\
\hline Interventricular septum [mm] & $0.88 \pm 0.08$ & $0.82 \pm 0.12$ & 0.36 \\
\hline Posterior wall $[\mathrm{mm}]$ & $0.89 \pm 0.07$ & $0.85 \pm 0.10$ & 0.46 \\
\hline Ejection fraction [\%] & $63.1 \pm 3.1$ & $61.8 \pm 2.8$ & 0.11 \\
\hline Left atrial volume $\left[\mathrm{mm}^{3}\right]$ & $38.1 \pm 13.2$ & $26.3 \pm 4.4$ & $<0.001$ \\
\hline Left atrial area $\left[\mathrm{mm}^{2}\right]$ & $18.2 \pm 4.0$ & $14.8 \pm 1.6$ & $<0.001$ \\
\hline \multicolumn{4}{|c|}{ Mitral posterior wall and TDI parameters } \\
\hline E velocity $[\mathrm{m} / \mathrm{s}]$ & $0.79 \pm 0.15$ & $0.77 \pm 0.11$ & 0.66 \\
\hline A velocity $[\mathrm{m} / \mathrm{s}]$ & $0.60 \pm 0.15$ & $0.61 \pm 0.09$ & 0.76 \\
\hline Deceleration time $[\mathrm{ms}]$ & $202 \pm 18.1$ & $203 \pm 17.0$ & 0.48 \\
\hline IVRT [ms] & $79.87 \pm 5.0$ & $79.78 \pm 5.0$ & 0.87 \\
\hline Mitral annulus $E^{\prime}[\mathrm{cm} / \mathrm{s}]$ & $13.55 \pm 3.75$ & $12.14 \pm 2.29$ & 0.09 \\
\hline Mitral annulus $A^{\prime}[\mathrm{cm} / \mathrm{s}]$ & $9.56 \pm 1.98$ & $8.54 \pm 1.87$ & 0.07 \\
\hline \multicolumn{4}{|l|}{ Atrial conduction times } \\
\hline $\mathrm{MS}[\mathrm{ms}]$ & $45.2 \pm 15.4$ & $34.6 \pm 7.2$ & $<0.001$ \\
\hline $\mathrm{ML}[\mathrm{ms}]$ & $66.9 \pm 18.2$ & $49.6 \pm 8.9$ & $<0.001$ \\
\hline $\mathrm{TL}[\mathrm{ms}]$ & $42.2 \pm 16.7$ & $37.5 \pm 6.9$ & 0.28 \\
\hline \multicolumn{4}{|c|}{ Atrial electromechanical delay times } \\
\hline ILeft-EMD (ML-MS) [ms] & $21.8 \pm 9.1$ & $14.1 \pm 4.9$ & $<0.001$ \\
\hline IRight-EMD (MS-TL) [ms] & $9.3 \pm 6.8$ & $5.9 \pm 4.9$ & 0.03 \\
\hline IA-EMD (ML-TL) [ms] & $24.7 \pm 11.2$ & $11.9 \pm 7.1$ & $<0.001$ \\
\hline
\end{tabular}

IA-EMD — inter-atrial electromechanical delay; ILeft-EMD — intra-left electromechanical delay; IRight-EMD — intra-right electromechanical delay; IVRT — isovolumetric relaxation time; LVEDd - left ventricular end diastolic diameter; LVESd - left ventricular end systolic diameter; MS — mitral septal; ML — mitral lateral; TL — tricuspid lateral; TDI — tissue Doppler imaging

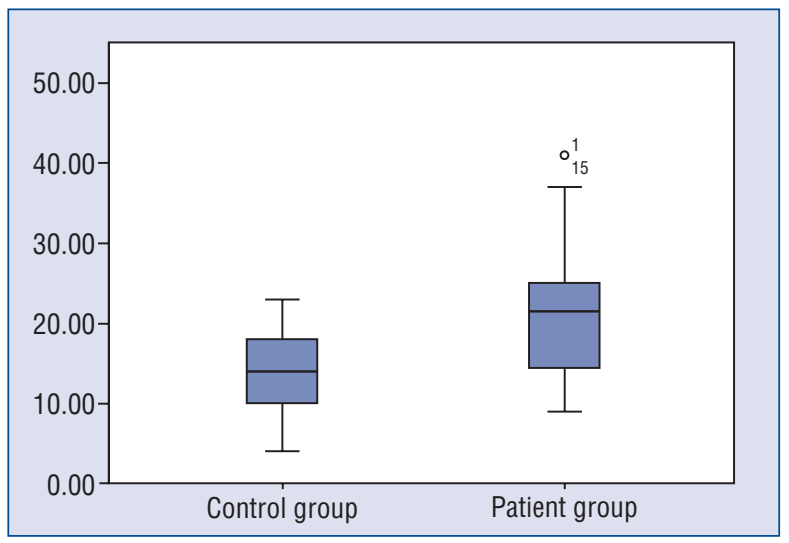

Figure 1. Comparison of intra-left atrial electromechanical delay times (ILeft-EMD)

was found to correlate with ILeft-EMD time $(\mathrm{r}=0.90, \mathrm{p}<0.001)$ and IA-EMD time $(\mathrm{r}=$ $=0.36, \mathrm{p}<0.004)$, whereas attack frequency did not correlate with the LA volume $(\mathrm{r}=0.12$, $\mathrm{p}<0.51)$, LA area $(\mathrm{r}=0.240, \mathrm{p}<0.18), \mathrm{E} / \mathrm{e}$ $(\mathrm{r}=0.08, \mathrm{p}<0.69), \mathrm{E} / \mathrm{A}(\mathrm{r}=-0.06, \mathrm{p}<0.73)$ or IRight-EMD time $(\mathrm{r}=-0.27, \mathrm{p}<0.13)$.

\section{Discussion}

The main findings of our study were that the ILeft-EMD time, IA-EMD time and LA volumes were significantly higher in the patient group. In addition, increased atrial conduction times measured from the mitral valve's lateral (ML) and septal annulus (MS) were detected in the patient group. In the patient group the annual frequency of $\mathrm{AF}$ attacks was found to be correlated with ILeft-EMD time and IA-EMD time.

It has been shown that focal discharges arising from pulmonary veins and posterior wall of LA have a major role in LAF pathogenesis [4]. In a current trial using the electrophysiological and electroanatomical mapping in LAF patients, shortening in atrial effective refractory period and conduction abnormalities characterized by prolongation of conduction times, longer P-wave durations, slower conduction velocities and an increase in the propor- 
tion of complex electrograms were detected [10]. Apart from the conditions that may contribute to abnormal atrial substrate such as fibrosis, inflammation, microvascular dysfunction and structural changes, the abovementioned electrical and structural changes constitute the additional factors that trigger AF progression [2]. Therefore, evaluating atrial conduction and electromechanical delay times may be predictive in patients with a high risk of $\mathrm{AF}$ development.

The patients' age, underlying heart disease, other medical conditions and echocardiographic findings are considered to determine the risk of $\mathrm{AF}$ development [2]. However, we need better markers to obtain earlier and more effective preventive measurements. Albeit the electrophysiological calculation is the gold standard method for calculation atrial conduction times, it is an invasive method not suitable for routine measurement in every patient.

Merkcx et al. [11] stated that the total atrial activation time can be determined by tissue Doppler technique, which may help detect patients under the risk of $\mathrm{AF}$ development. Consistent with our results, Weijs et al. [12] evaluated atrial conduction times of 41 idiopathic AF cases and 45 healthy individuals using the tissue Doppler echocardiography. In the idiopathic AF group, the total atrial conduction times were longer when compared to the control group with similar LA sizes.

Similar to our findings, a study comparing paroxysmal atrial fibrillation (PAF) patients with a control group, the only proven and significant independent variable in PAF patients was intra-left atrial conduction delay time. It was found that a $25 \mathrm{~ms}$ intra-left atrial conduction delay had a cutoff at $79 \%$ sensitivity, $66 \%$ specificity, $69 \%$ positive predictive and $76 \%$ negative predictive value [13].

In another study, using the tissue Doppler technique, atrial conduction times in patients with heart failure due to LV systolic dysfunction were found to be higher than the control group [14]. Yavuz et al. [15] proved that inter and intra-atrial electromechanical delay times increase in hypertensive patients with diastolic dysfunction. Since we didn't have any patients with LV systolic or diastolic dysfunction, we didn't determine a correlation between ejection fraction and echocardiographic intra-left and intra-right atrial conduction delay times.

In our study, we excluded patients with cardiopulmonary diseases which can cause LA dilation and pose a risk factor for $\mathrm{AF}$ such as hypertension, diabetes, rheumatic valvular diseases, diastolic or systolic heart failure and ischemic heart diseases.
The LA volumes and LA areas of LAF patients were found to be significantly higher than the control group and especially the atrial conduction times and electromechanical delay times were significantly higher in the patient group. LA remodeling could be the reason of ILeft-EMD and IA-EMD prolongation. These findings were completely compatible with the findings of previous electrophysiological findings, such as a shortened effective refractory period, conduction delays, conduction abnormalities characterized by area-specific conduction delays, slow conduction times and atrial dilation.

In our study, LAF patients were followed up for 1 year and their PAF attacks were recorded. As a result, although the conduction times measured from the mitral lateral annulus, ILeft-EMD and IA-EMD were found to be correlated with annual PAF attack frequencies, the same relationship was not observed with IRight-EMD, LA volume, LA area.

In the light of these findings, it can be thought that the increase in the atrial conduction times and electromechanical delay times would be one of the contributing factors to trigger and maintain AF. Our results are promising and future studies are needed to clarify this issue.

\section{Limitations of the study}

Our study was conducted in a single center with a small number of participants. The main limitation of our study was that conduction times were determined with tissue Doppler echocardiography and the gold standard technique, electrophysiological study, was not performed.

\section{Conclusions}

ILeft-EMD and IA-EMD may increase in the early stages of AF even without the LA dilation and may be more valuable than LA area and volume in predicting AF.

Conflict of interest: none declared

\section{References}

1. Freinberg WM, Blackshear JL, Laupacis A, Kronmal R, Hart RG. Prevalence, age distribution, and gender of patients with atrial fibrillation. Analysis and implications. Arch Intern Med, 1995; 155: 469-473.

2. Camm AJ, Kirchhof P, Lip GY et al. European Heart Rhythm Association; European Association for Cardio-Thoracic Surgery, ESC Committee for Practice Guidelines. Guidelines for the management of atrial fibrillation: the Task Force for the Management of Atrial Fibrillation of the European Society of Cardiology (ESC). Europace, 2010; 12: 1360-1342. 
3. Jahangir A, Lee V, Friedman PA et al. Long-term progression and outcomes with aging in patients with lone atrial fibrillation: A 30-year follow-up study. Circulation, 2007; 115: 3050-3056.

4. Haissaguerre M, Jais P, Shah DC et al. Spontaneous initiation of atrial fibrillation by ectopic beats originating in the pulmonary veins. N Engl J Med, 1998; 339: 659-666.

5. Cosio FG, Palacios J, Vidal JM, Cocina EG, Gomez-Sanchez MA, Tamargo L. Electrophysiologic studies in atrial fibrillation. Slow conduction of premature impulses: A possible manifestation of the background for reentry. Am J Cardiol, 1983; 51: 122-130.

6. Shimizu A, Fukatani M, Tanigawa M, Mori M, Hashiba K.. Intraatrial conduction delay and fragmented atrial activity in patients with paroxysmal atrial fibrillation. Jpn Circ J, 1989; 53: 1023-1030 .

7. Bayes de Luna A, Cladellas M et al. Interatrial conduction block and retrograde activation of the left atrium and paroxysmal supraventricular tachyarrhythmia. Eur Heart J, 1998; 9: 1112-1118.

8. Rein A, O'Donnel CP, Colan SD, Marx GR. Tissue velocity Doppler assessment of atrial and ventricular electromechanical coupling and atrioventricular time intervals in normal subjects. Am J Cardiol, 2003; 92: 1347-1350.

9. Lang RM, Bierig M, Devereux RB et al. Chamber Quantification Writing Group; American Society of Echocardiography's Guidelines and Standards Committee; European Association of Echocardiography. Recommendations for chamber quantification: a report from the American Society of Echocardiography's Guidelines and
Standards Committee and the Chamber Quantification Writing Group, developed in conjunction with the European Association of Echocardiography, a branch of the European Society of Cardiology. J Am Soc Echocardiogr, 2005; 18: 1440-1463.

10. Stiles MK, John B, Wong CX et al. Paroxysmal lone atrial fibrillation is associated with abnormal atrial substrate. Characterizing the "Second Factor". J Am Coll Cardiol, 2009; 53: 1182-1191.

11. Merckx KL, De Vos CB, Palmans A, Habets J, Cheriex EC, Crijns HJ, Tieleman RG. Atrial activation determined by transthoracic Doppler tissue imaging can be used as an estimate of the total duration of atrial electrical activation. J Am Soc Echocardiogr, 2005; 18: 940-944.

12. Weijs B, de Vos CB, Limantoro I, Cheriex EC, Tieleman RG, Crijns HJ. The presence of an atrial electromechanical delay in idiopathic atrial fibrillation as determined by tissue Doppler imaging. Int J Cardiol, 2012; 156: 121-122.

13. Deniz A, Yavuz B, Ciftci O et al. Left atrial conduction time detected by tissue Doppler imaging increases in paroxysmal atrial fibrillation patients. Clin Cardiol, 2006; 29 (suppl.): III-277.

14. Van Beeumen K, Duytschaever M, Tavernier R, Van de Veire N, De Sutter J. Intra- and interatrial asynchrony in patients with heart failure. Am J Cardiol, 2007; 99: 79-83.

15. Yavuz B, Abali G, Ciftci O et al. Atrial electromechanical delay gets longer in hypertensive patients with diastolic dysfunction. 2nd Annual Congress on Update in Cardiology and Cardiovascular Surgery. Clin Cardiol, 2006; 29 (suppl. 3): 21. 